\title{
Development and application of a forensic toxicological library for identification of 56 natural toxic substances by liquid chromatography-quadrupole time-of-flight mass spectrometry
}

\author{
Tadashi Ogawa $^{1,2} \cdot$ Kei Zaitsu $^{3} \cdot$ Tetsuo Kokaji $^{4} \cdot$ Kayako Suga $^{4} \cdot$ Fumio Kondo $^{1,5} \cdot$ Masae Iwai $^{1,2} \cdot$ Takayoshi Suzuki $^{1,2}$. \\ Akira Ishii ${ }^{3} \cdot$ Hiroshi Seno ${ }^{1,2}$
}

Received: 7 July 2019 / Accepted: 28 October 2019 / Published online: 17 November 2019

(c) The Author(s) 2019

\begin{abstract}
Purpose The present study aims to develop a forensic toxicological library to identify 56 natural toxic substances by liquid chromatography-quadrupole time-of-flight tandem mass spectrometry (LC-QTOF-MS/MS).

Methods For setting up the library of product ion spectra, individual substances ( 31 plant toxins, 7 mushroom toxins, 5 marine toxins, 5 frog venoms, 4 mycotoxins, and 4 substances derived from plants) were analyzed by LC-QTOF-MS/MS with positive and negative ionization. The product ion spectra were acquired at the collision energies (CEs) of 20, 35, and $50 \mathrm{eV}$ in single enhanced product ion mode and then in collision energy spread mode in which the CE ramp range was set to $35 \pm 15 \mathrm{eV}$.

Results To test the performance of the library, human blood plasma samples were spiked with a mixture of lycorine and domoic acid, extracted by acetonitrile deproteinization and analyzed by LC-QTOF-MS/MS. Identification by our library search could be achieved for these toxins at the purity scores of 79.1 and 67.2, respectively. The method was also applied to postmortem blood from a death case with an aconite intake, and showed that four toxins in an aconite could be identified in the blood sample at the purity scores of 54.6-60.3.

Conclusions This library will be more effective for the screening of natural toxic substances in routine forensic toxicological analysis. To our knowledge, there are no reports dealing with development of library for natural toxic substances by LC-QTOF-MS/MS.
\end{abstract}

Keywords Natural toxic substances · Forensic toxicological library $\cdot$ Screening and identification $\cdot$ Tetrodotoxin $\cdot$ Aconitine and amanitin $\cdot$ LC-QTOF-MS/MS

Electronic supplementary material The online version of this article (https://doi.org/10.1007/s11419-019-00506-w) contains supplementary material, which is available to authorized users.

Tadashi Ogawa

ogawatd@aichi-med-u.ac.jp

1 Department of Legal Medicine, Aichi Medical University School of Medicine, 1-1 Yazakokarimata, Nagakute 480-1195, Japan

2 Poison Analysis Center, Aichi Medical University School of Medicine, 1-1 Yazakokarimata, Nagakute 480-1195, Japan

3 Department of Legal Medicine and Bioethics, Nagoya University Graduate School of Medicine, 65 Tsurumai-cho, Showa-ku, Nagoya 466-8550, Japan

\section{Introduction}

Natural toxins are chemicals that are naturally produced by living organisms such as some plants, mushrooms, marine animals, and so on [1]. These toxins are not harmful to the

4 SCIEX, 4-7-35 Kitashinagawa, Shinagawa-ku, Tokyo 140-0001, Japan

5 Department of Biomedical Sciences, Chubu University College of Life and Health Sciences, 1200 Matsumoto-cho, Kasugai 487-8501, Japan 
organisms themselves but they may be toxic to other creatures, including humans, when eaten [1]. For example, tetrodotoxin in pufferfish and some marine animals is a powerful sodium channel blocker in excitable tissues such as nerves and muscles, and is about 10,000 times more lethal than cyanide by weight [2]. Forensic toxicology is a part of the pharmacological science, which is concerned with the identification/quantification and effects of various drugs and poisons in human beings [3]. Natural toxins are very important analytical targets in forensic toxicology [4-6]. It is difficult to attribute a cause of death to natural toxin(s) in routine toxicological analysis because there is currently no effective routine screening method for a variety of natural toxins [7].

In the last decade, liquid chromatography-tandem mass spectrometry (LC-MS/MS) has been developing its importance for the screening of drugs and/or poisons, most of which is based on triple quadrupole mass spectrometers employing multiple reaction monitoring survey scan followed by a product ion scan by electrospray ionization [8-11]. The application of this method is limited because of disturbances by high matrix burden and co-eluting peaks, indicating that analytes can be detected only if they are abundantly contained in the samples and that the method can easily lead to false positive/negative detection [12]. This drawback prompts the need for additional approaches achieving unambiguous identification of analytes.

Recently, liquid chromatography-quadrupole time-offlight tandem mass spectrometry (LC-QTOF-MS/MS) has been utilized to develop libraries of compounds relevant to clinical and forensic toxicology [13-21]. However, to the best of our knowledge, there are no reports on development of library for natural toxic substances by LC-QTOF-MS/ MS. In this paper, we describe the development and application of forensic toxicological library of 56 natural toxic substances using LC-QTOF-MS/MS.

\section{Materials and methods}

\section{Chemicals and reagents}

Target natural toxic substances were selected on the basis of previously reported poisoning cases as follows: 31 plant toxins (coniine, lycorine, galantamine, atropine, picrotoxinin, scopolamine, picrotin, strychnine, colchicine, veratramine, cyclopamine, jervine, amygdalin, aconine, cymarin, convallatoxin, cucurbitacin $\mathrm{E}$, oleandrin, benzoylmesaconine, benzoylaconine, tubocurarine, hypaconitine, mesaconitine, 14-anisoylaconine, aconitine, jesaconitine, digitoxin, digoxin, $\alpha$-chaconine, $\alpha$-solanine, and dioscin), 7 mushroom toxins (muscimol, ibotenic acid, muscarine, phalloidin, $\gamma$-amanitin, $\alpha$-amanitin, and $\beta$-amanitin), 4 mycotoxins (aflatoxin B1, aflatoxin B2, aflatoxin G1 and aflatoxin G2),
5 marine toxins (domoic acid, tetrodotoxin, okadaic acid, dinophysistoxin-1, and brevetoxin $b$ ), and 5 frog venoms (bufotenine, resibufogenin, bufalin, cinobufagin, and batrachotoxin). In addition to the natural toxins, berberine, cinchonidine, diosgenin, and quinine were selected as target substances, which are considered to be important materials of herbal medicines. Scopolamine, aflatoxin B1, aflatoxin B2, tetrodotoxin, quinine, aflatoxin G1, aflatoxin G2, resibufogenin, bufalin, colchicine, cyclopamine, diosgenin, cinobufagin, amygdalin, benzoylmesaconine, tubocurarine, mesaconitine, 14-anisoylaconine, jesaconitine, okadaic acid, and dinophysistoxin-1 were purchased from Fujifilm Wako Pure Chemical (Osaka, Japan); muscimol, coniine, ibotenic acid, muscarine, bufotenine, cinchonidine, strychnine, cymarin, convallatoxin, cucurbitacin E, oleandrin, digitoxin, digoxin, and $\alpha$-solanine from Sigma-Aldrich (St. Louis, MO, USA); picrotoxinin, picrotin, domoic acid, $\boldsymbol{\gamma}$-amanitin, and $\beta$-amanitin from Abcam Biochemicals (Cambridge, UK); berberine, aconine, and benzoylaconine from Cayman Chemical (Ann Arbor, MI, USA); galantamine, atropine, and jervine from Tokyo Chemical Industry (Tokyo, Japan); $\alpha$-chaconine and dioscin from Extrasynthese (Lyon, France); batrachotoxin and aconitine from Latoxan (Valence, France); phalloidin and $\alpha$-amanitin from Merck Millipore (Billerica, MA, USA); lycorine, hypaconitine, brevetoxin $b$, and veratramine from Enzo Life Sciences (New York, NY, USA); Kishida Chemical (Osaka, Japan), LKT Laboratories (St. Paul, MN, USA), and Toronto Research Chemicals (Toronto, Canada), respectively. The stock solutions of all substances were prepared at a concentration of $10-1000 \mu \mathrm{g} / \mathrm{mL}$. Muscarine, lycorine, cinchonidine, scopolamine, tetrodotoxin, quinine, berberine, resibufogenin, cinobufagin, amygdalin, hypaconitine, and $\alpha$-amanitin were dissolved in distilled water (DW). Aconitine and benzoylaconine were dissolved in acetonitrile. Other toxins were dissolved in methanol solution. Stock solutions were stored at $-80{ }^{\circ} \mathrm{C}$ until analysis. Methanol, acetonitrile and DW of the HPLC grade were purchased from Kanto Chemical (Tokyo, Japan). Other common chemicals used were of the highest purity commercially available. Human whole blood was obtained from Tennessee Blood Services (Memphis, TN, USA).

\section{LC-QTOF-MS (/MS) conditions}

Sciex Triple TOF 5600 mass spectrometer (Sciex, Framingham, MA, USA) and Shimadzu NexeraX2 LC system (Shimadzu Co., Kyoto, Japan) were used for analysis. The column used for chromatographic separation was L-column ODS $(150 \times 1.5 \mathrm{~mm}$ i.d., particle size $5.0 \mu \mathrm{m}$; Chemicals Evaluation and Research Institute, Sugito, Saitama, Japan). The column temperature was maintained at $40{ }^{\circ} \mathrm{C}$, and the gradient system was used with a mobile phase (A) $10 \mathrm{mM}$ ammonium formate in 5\% methanol aqueous solution and 
(B) $10 \mathrm{mM}$ ammonium formate in $95 \%$ methanol solution. Linear gradient elution was started from $100 \%$ A to $100 \%$ B over $15 \mathrm{~min}$. The $100 \% \mathrm{~B}$ was held for $5 \mathrm{~min}$. It was then returned to $100 \%$ A over $10 \mathrm{~min}$ for the next run. The autosampler was maintained at $4{ }^{\circ} \mathrm{C}$ and the injection volume was $10 \mu \mathrm{L}$. Electrospray ionization was used in both positive and negative modes. The optimal MS parameters were declustering potential at $80 \mathrm{~V}$ and information dependent acquisition (IDA) criteria set at over $50 \mathrm{cps}$. The LC-QTOF-MS system allowed the acquisition of highly sensitive full scan MS spectra with high resolution and mass accuracy. In addition, IDA can be used to collect MS/MS spectra for compound identification based on MS/MS library searching.

This LC-QTOF-MS (/MS) method had several advantages for accurate detection of natural toxic substances. For instance, the mass spectrometer used in this study, triple TOF 5600, had high throughput which enabled very fast MS/MS acquisition rates at as low as $20 \mathrm{~ms}$ accumulation time in IDA mode. To fully leverage the instrument speed and obtain the best depth of coverage, the IDA workflow was optimized such that software overhead is minimized. The IDA method consisted of a high-resolution TOF-MS survey scan could follow up to $50 \mathrm{MS} / \mathrm{MS}$ ions. The combined use of high-resolution MS and IDA were extremely effective for the simultaneous detection of natural toxic substances in forensic samples. The instrument gave the resolution of 35,000 .

Data acquisition and processing were performed by Analyst software and Peak View incorporated with the XIC Manager application (Sciex). The XIC Manager can be used for targeted processing of high-resolution MS and MS/MS data allowing for screening and identification with the highest confidence based on retention time (RT), mass error of molecular ion, isotopic pattern, and automatic MS/ MS library searching.

\section{Construction of library of natural toxic substances by LC-QTOF-MS (/MS)}

All target substances were analyzed to investigate their retention properties, isotopic ratios and high-resolution MS/MS spectra obtained by collision induced dissociation (CID) with the injected amount of each compound of $0.1 \mu \mathrm{g}$. The four spectra were acquired at the collision energy (CE) at 20,35 , and $50 \mathrm{eV}$ in single enhanced product ion (EPI) mode together with collision energy spread (CES) mode, in which the CE ramp range was set to $35 \pm 15 \mathrm{eV}$. The CES parameter, in conjunction with the $\mathrm{CE}$, determined the collision energy applied to the precursor ion in a product ion scan. The CE is ramped from low to high energies. The selection ranges of the precursor ion and RT of each compound for acquiring the library search were $20 \mathrm{mDa}$ and 4.0 min, respectively. Compound identification was based on chromatographic and mass spectrometric information, including RT error, mass error, isotope matching, and library search results. The product ion for library search could be chosen from four spectra by CID energies of $( \pm)$ 20, 35, 50, and $35 \pm 15 \mathrm{eV}$, automatically.

\section{Limits of detection and recovery rates}

To determine the limits of detection (LODs), 5 plots with different concentrations of each substance spiked into blank blood plasma were used. The LODs were defined as the concentrations giving a signal-to-ratio of $3: 1$. The recovery rates were calculated by the ratio of peak area obtained from a target substance spiked into ante-extraction matrix to that obtained from the substance spiked into post-extraction matrix.

\section{Analysis of spiked samples}

The blood plasma samples spiked with lycorine $(1 \mu \mathrm{g} / \mathrm{mL})$ and domoic acid $(10 \mu \mathrm{g} / \mathrm{mL})$ were prepared. A $100-\mu \mathrm{L}$ volume of blood plasma containing the target substances was mixed with $100 \mu \mathrm{L}$ methanol and $300 \mu \mathrm{L}$ acetonitrile. The mixture was then mixed by vortexing for $30 \mathrm{~s}$ and centrifuged at 15,000 $\mathrm{g}$ for $10 \mathrm{~min}$. The supernatant was transferred to another tube and evaporated with a centrifugal evaporator (CVE-2000; Tokyo Rikakikai, Tokyo, Japan). The residue was reconstituted in $100 \mu \mathrm{L}$ of $10 \mathrm{mM}$ ammonium formate in $5 \%$ methanol solution and mixed by vortexing for $1 \mathrm{~min}$. A $10-\mu \mathrm{L}$ of the extract solution was analyzed by LC-QTOF-MS/MS using our newly developed library.

\section{Application to forensic autopsy samples}

A 45-year-old male with groan was found at his home. He was taken to hospital by an ambulance, but died shortly afterward. Beside the body in the room, there were dried roots of an aconite plant. Femoral vein and right and left heart blood samples were collected at autopsy performed in our laboratory and stored at $-80{ }^{\circ} \mathrm{C}$ until analysis. The blood samples were treated and analyzed in the same way as spiked samples described above.

\section{Results}

\section{Development of library of natural toxic substances}

Registered data consisted of 56 natural toxic substances with compound name, source, formula, exact mass, polarity, exact mass of precursor ion, ion form, RT, LOD, and recovery rate (Table 1). Extracted ion chromatograms of simultaneous determination of 56 substances are shown in Fig. 1. Product 


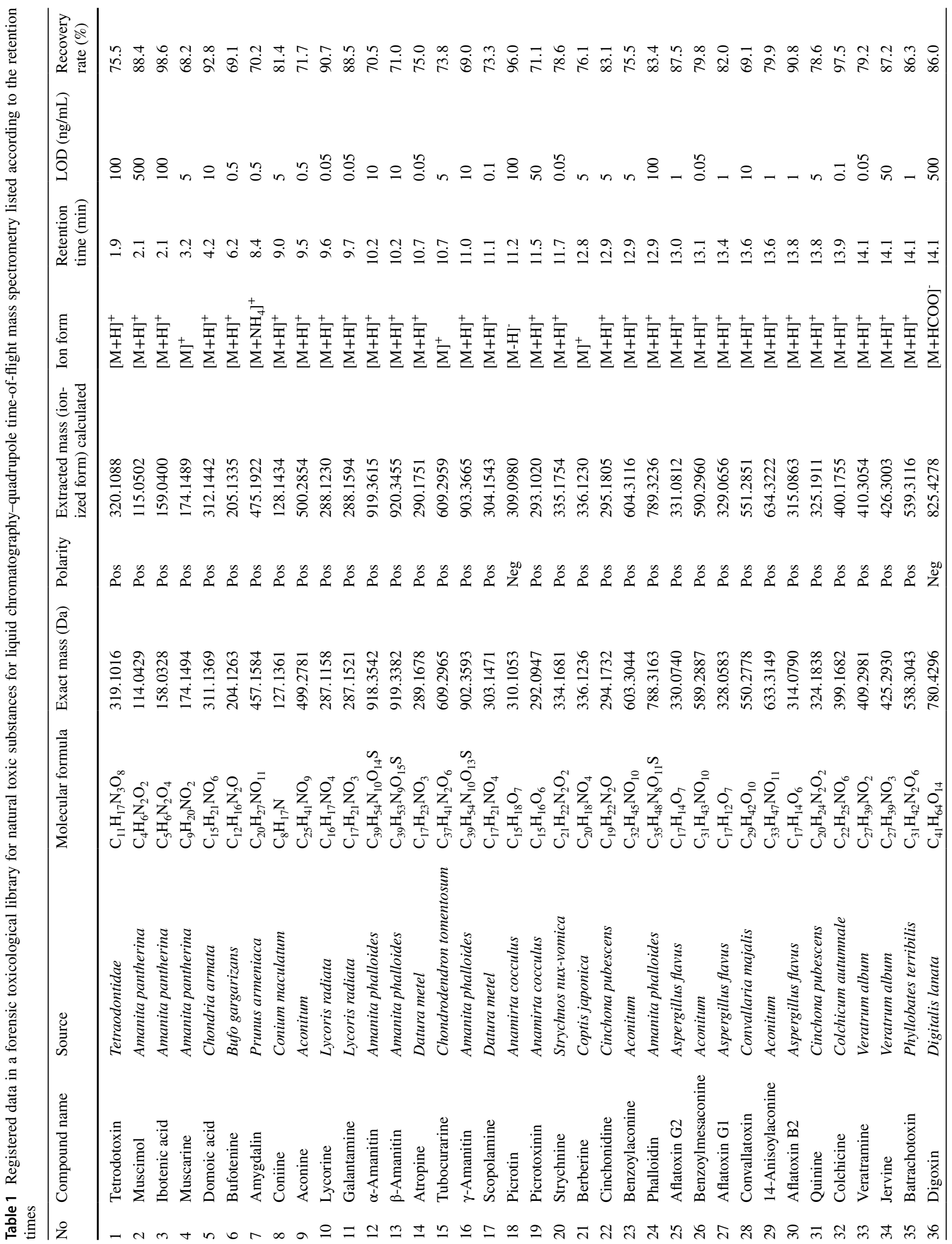




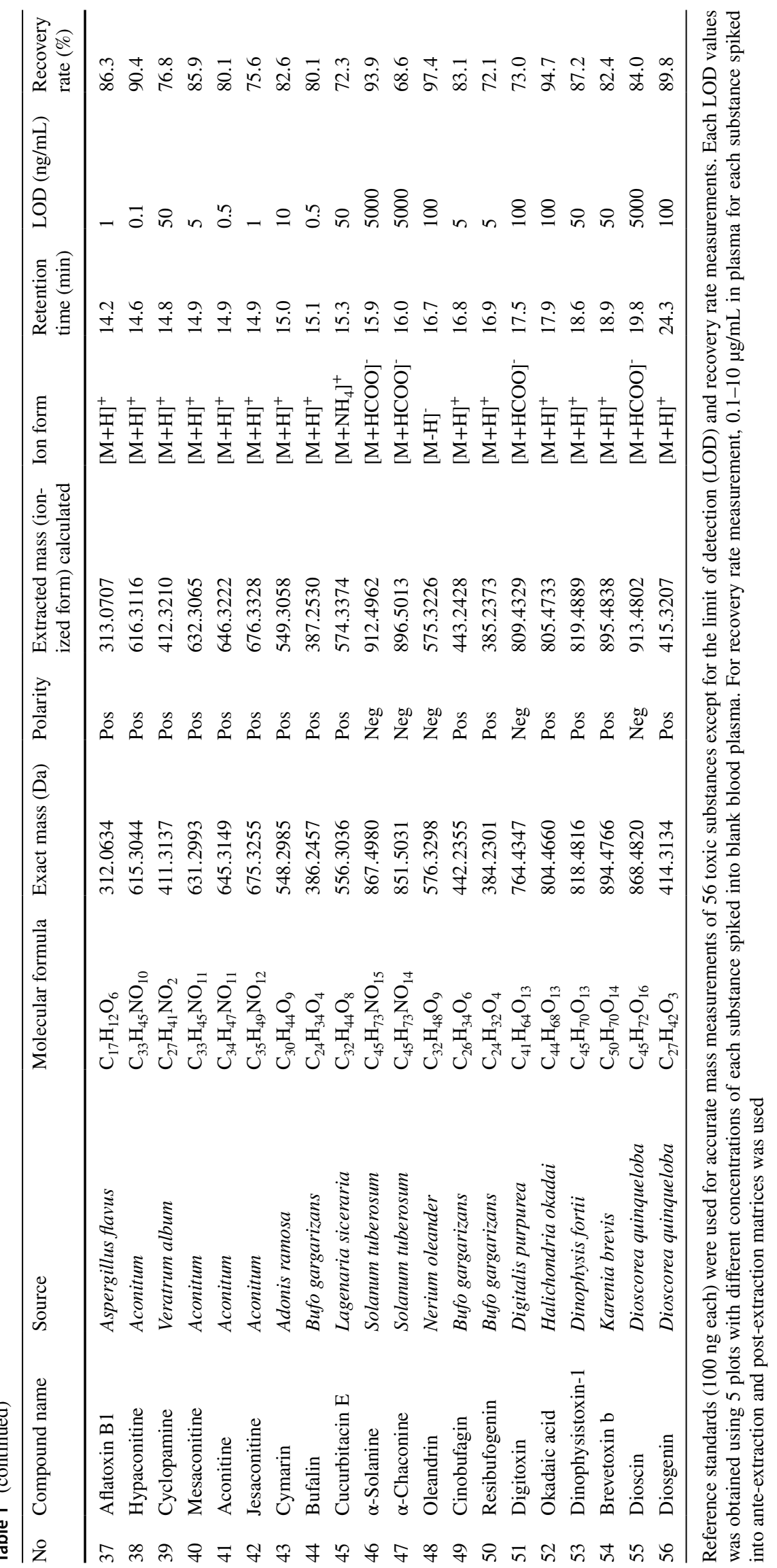




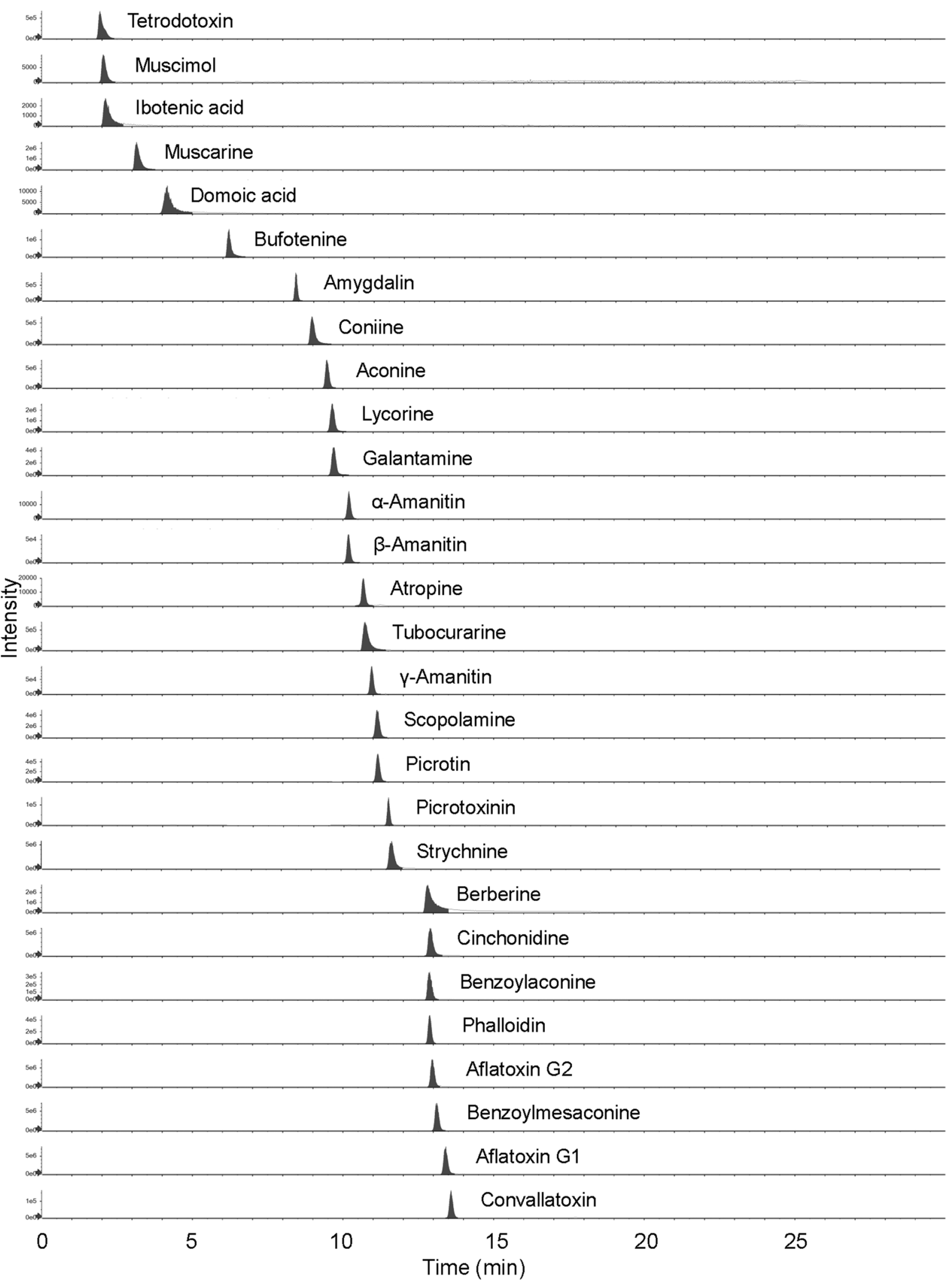

Fig. 1 Extracted ion chromatograms (XICs) of simultaneous determination of 56 natural toxic substances by liquid chromatography-quadrupole time-of-flight mass spectrometry (LC-QTOF-MS). The reference standards (100 ng each) were injected to the instrument 


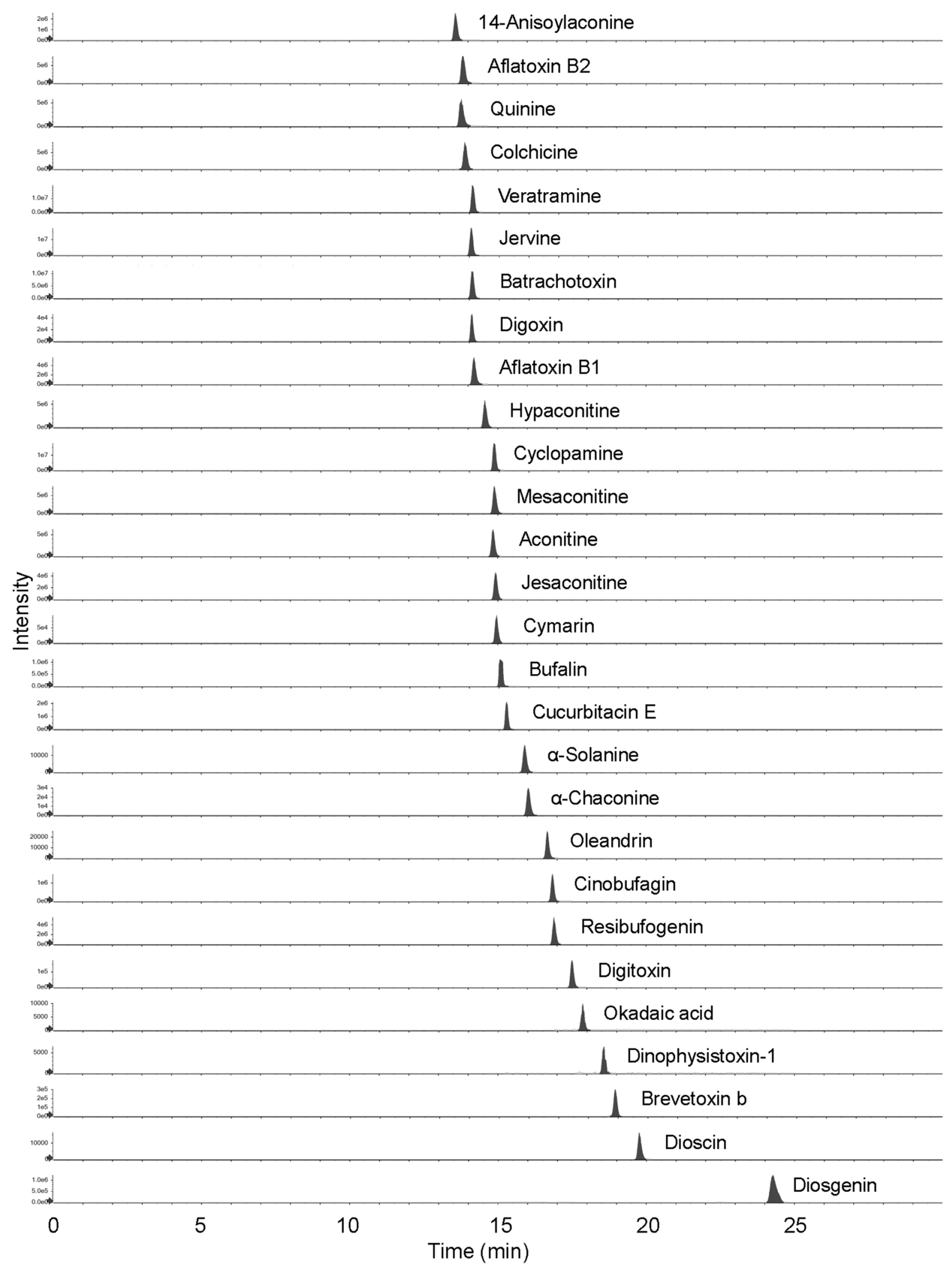

Fig. 1 (continued) 

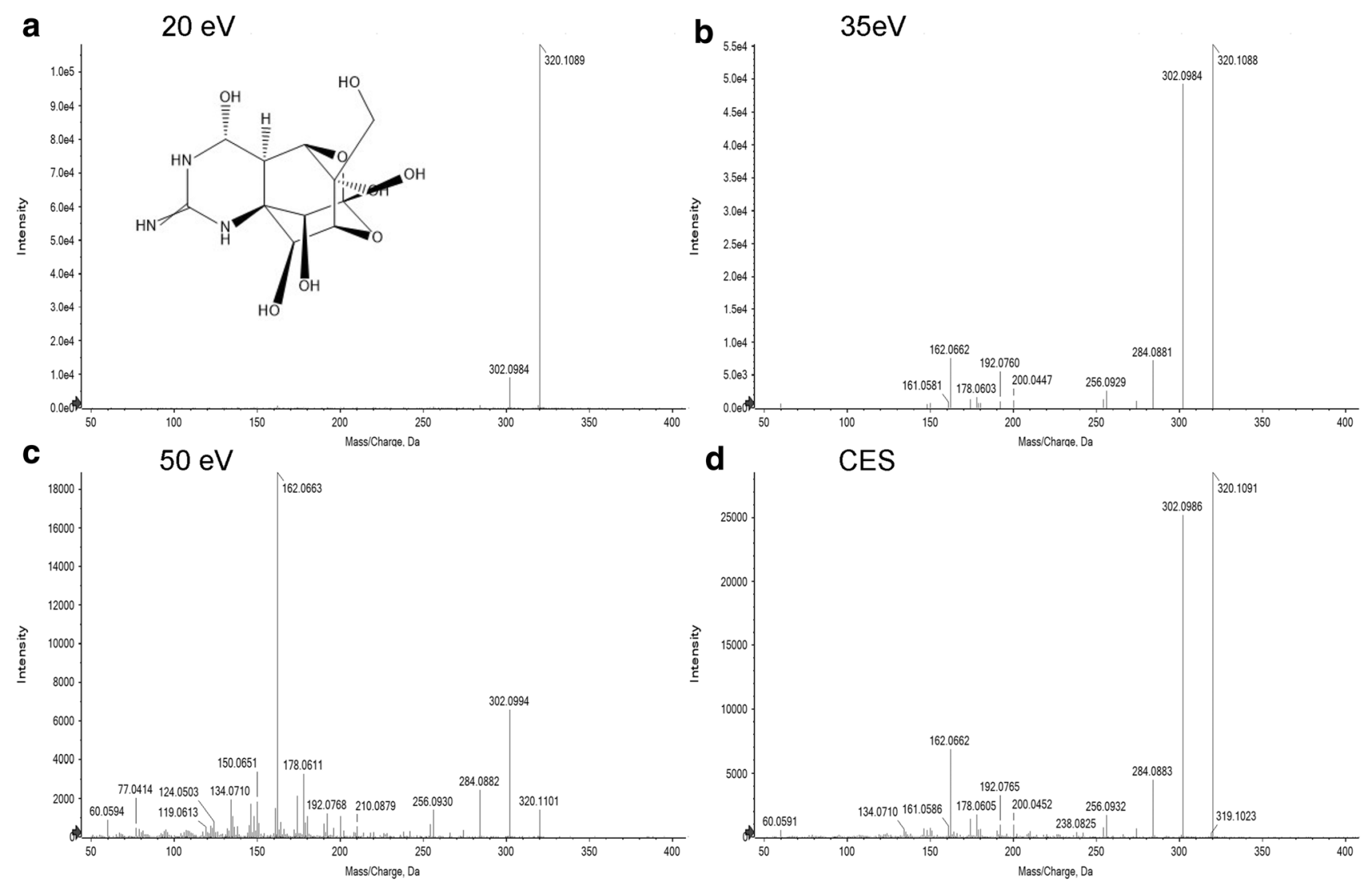

Fig. 2 Product ion spectra for tetrodotoxin at collision energy of a $20 \mathrm{eV}, \mathbf{b} 35 \mathrm{eV}, \mathbf{c} 50 \mathrm{eV}$, or d collision energy spread (CES), obtained by LCQTOF-MS/MS

ion spectra of all substances were obtained by four different CE settings (see supplementary material Fig. S1). As an example the four spectra obtained for tetrodotoxin, which is one of the important toxins in food poisoning cases in Japan, are shown in Fig. 2. The $[\mathrm{M}+\mathrm{H}]^{+}(\mathrm{m} / z$ 320.1088) was the most abundant ion at $20 \mathrm{eV}$ (Fig. 2a), while it remarkably decreased at $35 \mathrm{eV}$ (Fig. 2b) and became a very small peak at $50 \mathrm{eV}$ (Fig. 2c), and the number and intensity of fragment ions increased instead (Fig. 2a-c). Product ion spectra obtained by CES mode showed both $[\mathrm{M}+\mathrm{H}]^{+}$and fragment ions (Fig. 2d). CES mode can collect an average MS spectrum of different $\mathrm{CE}$ values in one single EPI scan, resulting in a full scan spectrum with both molecular and fragment ion information that can be used in library search-based identification with increased confidence. Digoxin, $\alpha$-solanine, $\alpha$-chaconine, digitoxin and dioscin provided $\left[\mathrm{M}+\mathrm{HCOO}^{-}\right.$, and amygdalin and cucurbitacin $\mathrm{E}$ showed $\left[\mathrm{M}+\mathrm{NH}_{4}\right]^{+}$ instead of $[\mathrm{M}+\mathrm{H}]^{+}$(Table 1 , supplementary material Fig. S1, nos. 36, 46, 47, 51, 57, 7, and 45, respectively); therefore, it is necessary to pay attention to this phenomenon.

Several precursor ions accompany unknown ions with strange mass defects. For example, the $m / z$ of $[\mathrm{M}+\mathrm{H}]^{+}$of strychnine (compounds no. 20) is 335.1754 , but 6 ions were observed in the range of 335-337 (336.2 could be the isotopic ion) (supplementary material Fig. S1, no. 20). This is also observed in the spectra of berberine (no. 21), aflatoxin G1 (no. 27), colchicine (no. 32), veratramine (no. 33), jervine (no. 34), aflatoxin B1 (no. 37), cyclopamine (no. 39), and Aconitum alkaloids (nos. 9, 23, 26, 29, 38, and 40-42). Unfortunately, the sources of the ions are still unknown. In future, it is necessary to analyze the assignment of these ions.

With respect to the chromatographic separation, water/ methanol both containing $10 \mathrm{mM}$ ammonium formate was used. The used conditions provided RTs ranging from $1.9 \mathrm{~min}$ to $24.3 \mathrm{~min}$ (Table 1). Although some hydrophilic substances like ibotenic acid, musimol, and muscarine eluted quickly, it had no problems to detect them by high-resolution MS analysis.

\section{Analysis of spiked samples}

The present library was applied for the identification of lycorine and domoic acid spiked into blank blood plasma. 
a XIC: Lycorine, 288.1230 Da

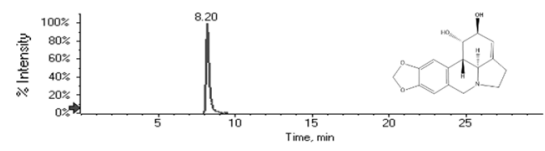

b XIC: Domoic acid, $312.1442 \mathrm{Da}$

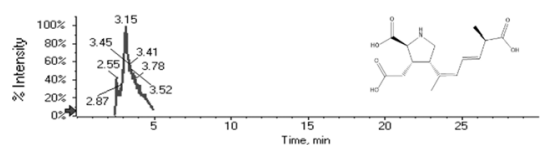

TOF-MS spectrum from 8.183-8.209 min

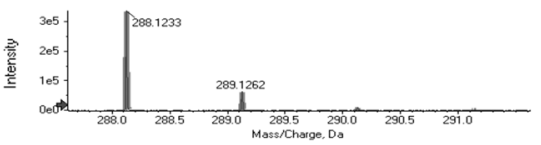

TOF-MS spectrum from 3.144-3.170 min

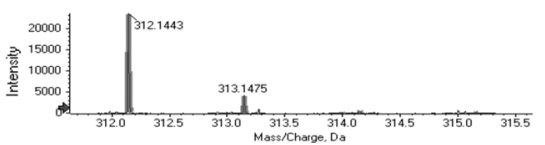

TOF-MS/MS spectrum from $8.266 \mathrm{~min}$ Precursor: 288.1 Da, CE: $35 \pm 15 \mathrm{eV}$

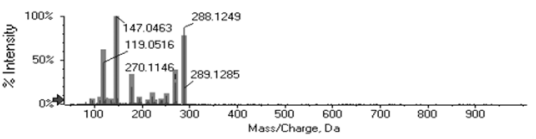

TOF-MS/MS spectrum from $3.204 \mathrm{~min}$ Precursor: $312.1 \mathrm{Da}, \mathrm{CE}: 35 \pm 15 \mathrm{eV}$

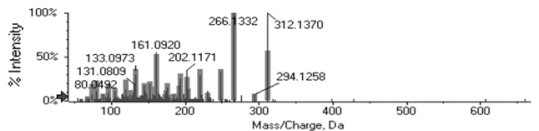

Fig. 3 Identification of lycorine $(1 \mu \mathrm{g} / \mathrm{mL})$ and domoic acid $(10 \mu \mathrm{g} / \mathrm{mL})$ spiked into blank blood plasma by LC-QTOF-MS/MS. XICs (left panels), TOF-MS spectra (100-1000 Da, middle panels) and TOF-MS/MS spectra (50-1000 Da, right panels) of a lycorine and $\mathbf{b}$ domoic acid

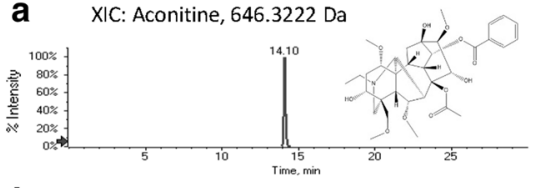

b XIC: Jesaconitine, $676.3328 \mathrm{Da}$

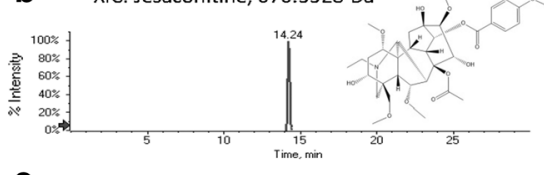

C XIC: Hypaconitine, 616.3116 Da

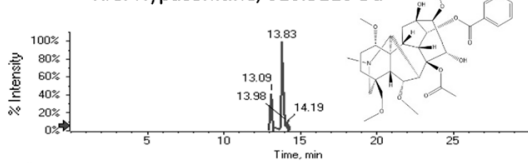

d XIC: Mesaconitine, 632.3065 Da

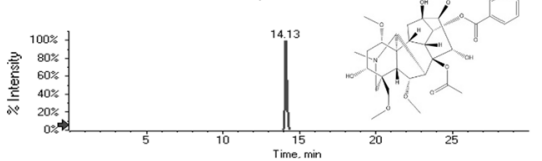

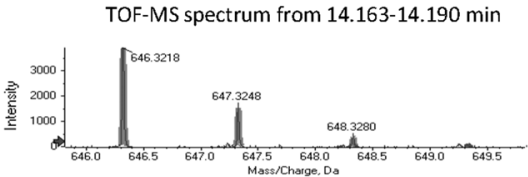

TOF-MS spectrum from 14.228-14.254 min

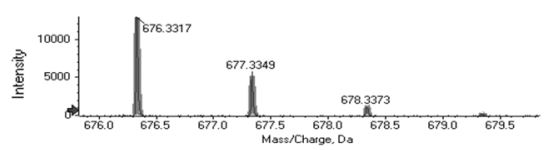

TOF-MS spectrum from $13.900-13.926 \mathrm{~min}$

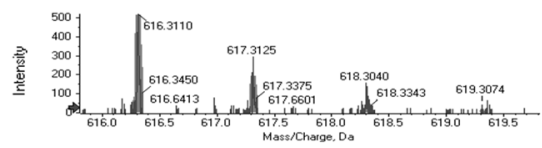

TOF-MS spectrum from 14.163-14.190 $\mathrm{min}$

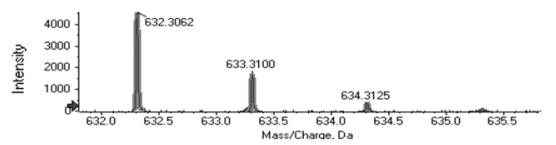

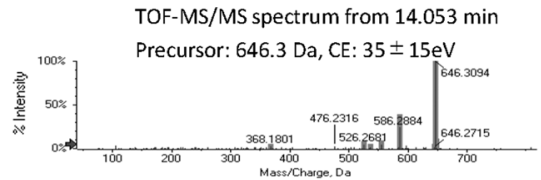

TOF-MS/MS spectrum from $14.158 \mathrm{~min}$

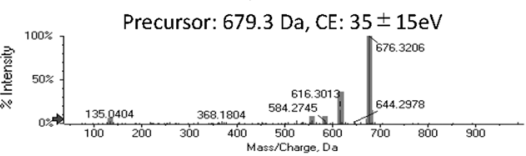

TOF-MS/MS spectrum from $13.844 \mathrm{~min}$ Precursor: 616.3 Da, CE: $35 \pm 15 \mathrm{eV}$

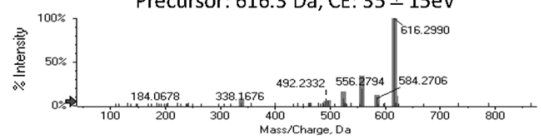

TOF-MS/MS spectrum from $14.079 \mathrm{~min}$ Precursor: $632.3 \mathrm{Da}, \mathrm{CE}: 35 \pm 15 \mathrm{eV}$

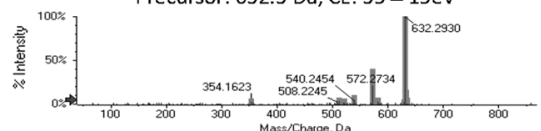

Fig. 4 Identification of aconitine, jesaconitine, hypaconitine, and mesaconitine in a forensic autopsy sample by LC-QTOF-MS/MS. XICs (left panels), TOF-MS spectra (100-1000 Da, middle panels), and TOF-MS/MS spectra (50-1000 Da, right panels) of a aconitine, $\mathbf{b}$ jesaconitine, $\mathbf{c}$ hypaconitine, and $\mathbf{d}$ mesaconitine

performed in our laboratory were analyzed using the present library. In all samples, aconitine, jesaconitine, hypaconitine, and mesaconitine were identified, and Fig. 4 shows representative results from the femoral vein sample. In the previous study, Niitsu et al. [22] reported the four poisons as major substances detected from blood in the cases of suicide by aconite poisoning. In the TOF-MS spectra, the measured masses matched the registered masses with mass errors of 0.5-1.0 ppm. In the TOF-MS/MS spectra, the masses of the fragment ions agree very well with the registered MS/MS spectra. The purity score was 54.6-60.3\%.

\section{Application to forensic autopsy samples}

Femoral vein and right and left heart blood samples collected from a 45-year-old male at the forensic autopsy 


\section{Discussion}

In this article, we have created a forensic toxicological library including 56 natural toxic substances. The drugs of abuse originated from plants, such as $\Delta^{9}$ tetrahydrocannabinol and cocaine have been excluded. To our knowledge, only one trial to construct libraries specific to natural toxic substances has been published [6]; but they used low-resolution LC-MS/MS instrument unable to make estimation of the molecular formulae, using its accurate mass numbers, which are very useful for tentative identification of an unknown substances. Recently, Wang et al. [23] reported high-throughput screening of more than 200 toxic substances including narcotic drugs, psychotropic drugs, pesticides, natural toxins, and other drugs; however, in their collection, only 3 substances were in common with those in our article. Moreover, they did not use a high-resolution MS instrument, but a low-resolution linear ion trap quadrupole MS coupled with a homemade extractive electrospray ionization. Broecker et al. [12] reported an article on development and application of a library for CID accurate mass spectra of more than 2500 toxic compounds by LC-QTOFMS/MS. However, the readers cannot get access to the MS/ MS spectra only with their paper. In the present article, the readers can readily gain access to the detailed high-resolution MS/MS spectra of 56 natural toxic substances located in the electronic supplementary material.

Martin et al. [24] compared the performance of three types of LC-QTOF-MS/MS platforms created by three different manufacturers including the Sciex Triple TOF 5600 system used in the present study. There are usually three parameters for compound identification by LC-QTOF-MS/ MS: mass errors not greater than 4 or $5 \mathrm{ppm}$, RT differences with $0.2-0.5 \mathrm{~min}$, and similarities of MS/MS spectrum profiles. The former two parameters were common to the three types of the instruments. For the similarity of the MS/ MS profiles, one manufacturer did not incorporate such a parameter as of 2014. Another manufacturer provided MS/ MS libraries at three collision energies for matching. The system of our instrument takes into consideration the presence/absence of all MS/MS spectral peaks and their relative abundance, which are compared to those of the MS/MS library record, calculating the purity score. In addition, the system also includes the CES mode, in which the CE ramp range is set to $35 \pm 15 \mathrm{eV}$, in which a small parent peak and important small product ions are magnified automatically. Therefore, we presented three MS/MS spectra at CEs of 20, 35 , and $50 \mathrm{eV}$, and one spectrum in the CES mode (Fig. 2, Fig. S1). Such algorithms adopted by Sciex for comparison of MS/MS spectrum profiles seem most sophisticated and thus reliable in current LC-QTOF-MS arena. Although some previous studies described identification of target compounds using the purity scores, their distinct criteria have not been established [16, 25-27]. According to our results on the spiked and forensic autopsies (Figs. 3, 4), the purity scores more than $50 \%$ seems to be acceptable prior to considering the matches of a mass error and RT.

When the present library of natural toxic substances by LC-QTOF-MS were created, low-resolution MS/MS spectra of 54 natural toxic substances were also recorded, except for picrotoxinin and diosgenin (unpublished observation). The low-resolution MS/MS spectra at CEs of 20, 35 , and $50 \mathrm{eV}$, and one spectrum in the CES mode were acquired; the low-resolution MS/MS spectra were similar to the high-resolution MS/MS spectra in this study. Therefore, the detailed high-resolution MS/MS spectra of natural toxic substances located in the electronic supplementary material in the present article (Fig. 2, Fig. S1) seems to be also useful in routine forensic toxicological screening by low-resolution LC-MS/MS.

\section{Conclusions}

We have developed a forensic toxicological library for identification of 56 natural toxic substances by LC-QTOF-MS/ MS. The applicability of the library was exemplified by identifying four plant toxins in blood samples collected from an autopsy. This library may be effective for the screening of natural toxic substances and can become a powerful tool for searching natural toxic substances in routine forensic toxicological analysis. To our knowledge, this is the first trial to develop a toxicological library for natural toxic substances using high-resolution LC-MS/MS.

Acknowledgements This work was supported by JSPS KAKENHI (Grant no. 18H03064).

\section{Compliance with ethical standards}

Conflict of interest The authors declare that they have no conflict of interest.

Ethical approval The analysis of blood samples from deceased subjects was requested by the judicial authorities.

Open Access This article is distributed under the terms of the Creative Commons Attribution 4.0 International License (http://creativeco mmons.org/licenses/by/4.0/), which permits unrestricted use, distribution, and reproduction in any medium, provided you give appropriate credit to the original author(s) and the source, provide a link to the Creative Commons license, and indicate if changes were made. 


\section{References}

1. WHO (2018) Natural toxins in food. https://www.who.int/newsroom/fact-sheets/detail/natural-toxins-in-food. Accessed $27 \mathrm{Dec}$ 2018

2. Leung KS, Fong BM, Tsoi YK (2011) Analytical challenges: determination of tetrodotoxin in human urine and plasma by LC-MS/MS. Mar Drugs 9:2291-2303. https://doi.org/10.3390/ md9112291

3. Smith MP, Bluth MH (2016) Forensic toxicology: an introduction. Clin Lab Med 36:753-759. https://doi.org/10.1016/j. cll.2016.07.002

4. Drummer OH (2018) Toxicology: overview and applications. In: Houch MM (ed) Forensic Toxicology. Academic press, London, pp 25-31

5. Hasegawa K, Gonmori K, Fujita H, Kamijo Y, Nozawa H, Yamagishi I, Minakata K, Watanabe K, Suzuki O (2013) Determination of ibotenic acid and muscimol, the Amanita mushroom toxins, in human serum by liquid chromatography-tandem mass spectrometry. Forensic Toxicol 31:322-327. https://doi.org/10.1007/s1141 9-013-0189-2

6. Zeng Y, Quek Y-L, Kee C-L, Low M-Y, Bloodworth BC, Ge X (2015) Analysis of 32 toxic natural substances in herbal products by liquid chromatography quadrupole linear ion trap mass spectrometry. J Pharm Biomed Anal 115:169-173. https://doi. org/10.1016/j.jpba.2015.07.005

7. Smith ML, Vorce SP, Holler JM, Shimomura E, Magluilo J, Jacobs AJ, Huestis MA (2007) Modern instrumental methods in forensic toxicology. J Anal Toxicol 31:237-253. https://doi. org $/ 10.1093 / \mathrm{jat} / 31.5 .237$

8. Maurer HH (2004) Position of chromatographic techniques in screening for detection of drugs or poisons in clinical and forensic toxicology and/or doping control. Clin Chem Lab Med 42:1310 1324. https://doi.org/10.1515/CCLM.2004.250

9. Maurer HH (2007) Current role of liquid chromatographymass spectrometry in clinical and forensic toxicology. Anal Bioanal Chem 388:1315-1325. https://doi.org/10.1007/s0021 6-007-1248-5

10. Remane D, Wissenbach DK, Peters FT (2016) Advances of liquid chromatography-(tandem) mass spectrometry in clinical and forensic toxicology—an update. Clin Biochem 49:1051-1071. https://doi.org/10.1016/j.clinbiochem.2016.07.010

11. Mbughuni MM, Jannetto PJ, Langman LJ (2016) Mass spectrometry applications for toxicology. EJIFCC 27:272-287. https://www. ncbi.nlm.nih.gov/pmc/articles/PMC5282913/

12. Broecker S, Herre S, Wüst B, Zweigenbaum J, Pragst F (2011) Development and practical application of a library of CID accurate mass spectra of more than 2,500 toxic compounds for systematic toxicological analysis by LC-QTOF-MS with datadependent acquisition. Anal Bioanal Chem 400:101-117. https ://doi.org/10.1007/s00216-010-4450-9

13. Tyrkkö E, Pelander A, Ojanperä I (2010) Differentiation of structural isomers in a target drug database by LC/Q-TOFMS using fragmentation prediction. Drug Test Anal 2:259-270. https://doi. org/10.1002/dta.134

14. Campbell JL, Le Blanc JCY (2012) Using high-resolution quadrupole TOF technology in DMPK analyses. Bioanalysis 4:487-500. https://doi.org/10.4155/bio.12.14

15. Guale F, Shahreza S, Walterscheid JP, Chen HH, Arndt C, Kelly AT, Mozayani A (2013) Validation of LC-TOF-MS screening for drugs, metabolites, and collateral compounds in forensic toxicology specimens. J Anal Toxicol 37:17-24. https://doi.org/10.1093/ jat/bks084

16. Herrera-Lopez S, Hernando MD, García-Calvo E, FernándezAlba AR, Ulaszewska MM (2014) Simultaneous screening of targeted and non-targeted contaminants using an LC-QTOF-MS system and automated MS/MS library searching. J Mass Spectrom 49:878-893. https://doi.org/10.1002/jms.3428

17. Kildgaard S, Mansson M, Dosen I, Klitgaard A, Frisvad JC, Larsen TO, Nielsen KF (2014) Accurate dereplication of bioactive secondary metabolites from marine-derived fungi by UHPLCDAD-QTOFMS and a MS/HRMS library. Mar Drugs 12:36813705. https://doi.org/10.3390/md12063681

18. Bussche JV, Decloedt A, Van Meulebroek L, De Clercq N, Lock S, Stahl-Zeng J, Vanhaecke L (2014) A novel approach to the quantitative detection of anabolic steroids in bovine muscle tissue by means of a hybrid quadrupole time-of-flight-mass spectrometry instrument. J Chromatogr A 1360:229-239. https://doi. org/10.1016/j.chroma.2014.07.087

19. Paul M, Ippisch J, Herrmann C, Guber S, Schultis W (2014) Analysis of new designer drugs and common drugs of abuse in urine by a combined targeted and untargeted LC-HR-QTOFMS approach. Anal Bioanal Chem 406:4425-4441. https://doi.org/10.1007/ s00216-014-7825-5

20. Fels H, Dame T, Sachs H, Musshoff F (2017) Liquid chromatography-quadrupole-time-of-flight mass spectrometry screening procedure for urine samples in forensic casework compared to gas chromatography-mass spectrometry. Drug Test Anal 9:824-830. https://doi.org/10.1002/dta.2039

21. Liu H-C, Yang C-A, Liu RH, Lin D-L (2017) Developing a UHPLC-QTOF-MS and automated library search method for screening drugs and toxic compounds in postmortem specimens. J Anal Toxicol 41:421-430. https://doi.org/10.1093/jat/bkx026

22. Niitsu H, Fujita Y, Fujita S, Kumagai R, Takamiya M, Aoki Y, Dewa K (2013) Distribution of Aconitum alkaloids in autopsy cases of aconite poisoning. Forensic Sci Int 227:111-117. https ://doi.org/10.1016/j.forsciint.2012.10.021

23. Wang S, Li F, Liu Y, Zhao H, Chen H (2019) High-throughput screening of toxic substances by extractive electrospray ionization mass spectrometry and their identification via databank construction. Anal Bioanal Chem 411:4049-4054. https://doi.org/10.1007/ s00216-018-1520-x

24. Marin SJ, Sawyer JC, He X, Johnson-Davis KL (2015) Comparison of drug detection by three quadrupole time-of-flight mass spectrometry platforms. J Anal Toxicol 39:89-95. https://doi. org/10.1093/jat/bku134

25. Gómez MJ, Gómez-Ramos MM, Malato O, Mezcua M, Férnandez-Alba AR (2010) Rapid automated screening, identification and quantification of organic micro-contaminants and their main transformation products in wastewater and river waters using liquid chromatography-quadrupole-time-of-flight mass spectrometry with an accurate-mass database. J Chromatogr A 1217:70387054. https://doi.org/10.1016/j.chroma.2010.08.070

26. Martínez Bueno MJ, Ulaszewska MM, Gomez MJ, Hernando MD, Fernández-Alba AR (2012) Simultaneous measurement in mass and mass/mass mode for accurate qualitative and quantitative screening analysis of pharmaceuticals in river water. J Chromatogr A 1256:80-88. https://doi.org/10.1016/j.chroma.2012.07.038

27. Masiá A, Campo J, Blasco C, Picó Y (2014) Ultra-high performance liquid chromatography-quadrupole time-of-flight mass spectrometry to identify contaminants in water: an insight on environmental forensics. J Chromatogr A 1345:86-97. https:// doi.org/10.1016/j.chroma.2014.04.017

Publisher's Note Springer Nature remains neutral with regard to jurisdictional claims in published maps and institutional affiliations. 